\title{
The analytical solution of the 3D model with Robin's boundary conditions for 2 peat layers
}

\author{
Ērika Teirumnieka ${ }^{1}$, Ilmārs Kangro ${ }^{1}$, Edmunds Teirumnieks ${ }^{1}$, Harijs Kalis ${ }^{2}$ \\ ${ }^{1}$ Rezekne Higher Education Institution, \\ Atbrīvošanas aleja 115, LV-4601, Rēzekne, Latvia, \\ Phone: +371 4625150, Fax +371 4625901; \\ e-mail:Erika.Teirumnieka@ru.lv,ilmars.kangro@ru.lv,Edmunds.Teirumnieks@ru.lv \\ ${ }^{2}$ Institute of Mathematics, Latvian Academy of Sciences and University of Latvia, \\ Zelllu 8, Riga LV-1002, Latvia, \\ Phone+37167033721,Fax+37167820113; e-mail:kalis@lu.lv
}

\begin{abstract}
In this paper we consider averaging methods for solving the 3-D boundary value problem in domain containing 2 layers of the peat block. We consider the metal concentration in the peat blocks. Using experimental data the mathematical model for calculation of concentration of metal in different points in every peat layer is developed. A specific feature of these problems is that it is necessary to solve the 3-D boundary-value problems for elliptic type partial differential equations of second order with piece-wise diffusion coefficients in every direction and peat layers.

The special parabolic and exponential spline, which interpolation middle integral values of piece-wise smooth function, are considered. With the help of this splines is reduce the problems of mathematical physics in 3-D with piece-wise coefficients to respect one coordinate to problems for system of equations in 2-D. This procedure allows reduce the 3-D problem to a problem of 2-D and 1-D problems and the solution of the approximated problem is obtained analytically.

The solution of corresponding averaged 2-D initial-boundary value problem is obtained also numerically, using for approach differential equations the discretization in space applying the central differences. The approximation of the 2-D non-stationary problem is based on the implicit finitedifference and alternating direction (ADI) methods. The numerical solution is compared with the analytical solution.
\end{abstract}

Keywords: diffusion problem, special splines, analytical and numerical solution.

\section{INTRODUCTION}

The task of sufficient accuracy numerical simulation of quickly solution 3-D problems for mathematical physics in multilayered media is important in known areas of the applied sciences. To achieve this goal we consider two methods: special finite difference scheme and averaging method by using integral parabolic and exponential splines. For engineering calculation the concentration of metals in peat layered blocks is chosen the averaging method.

The finite-difference method is used only for solving the obtained 1-D and 2-D problems. The layered peats block are modelled in [5], [4].

A. Buikis ([1], [3]) considered different assumptions for averaging methods along the vertical coordinate. These methods were applied for the mathematical simulation of the mass transfer process in multilayered underground systems. It is necessity to solve the 3-D initial-boundary-value problems for parabolic type partial differential equations of second order with piece-wise parameters in multilayer domain. The special spline, which interpolation middle integral values of piece-wise smooth function, is defined. With the help of this splines is reduce the problems of mathematical physics in 3-D with piecewise coefficients to respect one coordinate analytically to problems for system of equations in 2-D.

The solutions of corresponding 2-D initialboundary value problem are obtained numerically, using the implicit finite difference approximation and 
alternating method of Douglas and Rockford. The 3-D problem is reduced to 2-D and 1-D problems used integral parabolic and exponential splines.

\section{MATHERIALS AND METHODS}

1. A mathematical model

The process of diffusion the metal in the peat block is consider in 3-D parallelepiped

$\Omega=\left\{(x, y, z): 0 \leq x \leq L_{x}, 0 \leq y \leq L_{y}, 0 \leq z \leq L_{z}\right\}$

The domain $\Omega$ consists of multilayer medium. We will consider the non-stationary 3-D problem of the linear diffusion theory for multilayered piece-wise homogenous materials of 2 layers in the form $\Omega_{i}=\left\{(x, y, z): x \in\left(0, L_{x}\right), y \in\left(0, L_{y}\right), z \in\left(z_{i-1}, z_{i}\right)\right\}, i=1 ; 2$

where $H_{i}=z_{i}-z_{i-1}$ is the height of layer $\Omega_{i}$, $z_{0}=0, z_{2}=L_{z}$.

We will find the distribution of concentrations $c_{i}=c_{i}(x, y, z, t)$ in every layer $\Omega_{i}$ at the point $(x, y, z) \in \Omega_{i}$ and at the time $t$ by solving the following 3-D initial-boundary value problem for partial differential equation (PDE):

$$
\begin{aligned}
& \frac{\partial c_{i}(x, y, z, t)}{\partial t}=\frac{\partial}{\partial x}\left(D_{i x} \frac{\partial c(x, y, z, t)}{\partial x}\right)+\frac{\partial}{\partial y}\left(D_{i y} \frac{\partial c(x, y, z, t)}{\partial y}\right)+ \\
& \frac{\partial}{\partial z}\left(D_{i z} \frac{\partial c(x, y, z, t)}{\partial z}\right)+f_{i}(x, y, z, t) \\
& x \in\left(0, L_{x}\right), y \in\left(0, L_{y}\right), z \in\left(0, L_{z}\right), \\
& t \in\left(0, t_{f}\right), i=1 ; 2 \\
& \partial c_{i}(0, y, z, t) / \partial x=\partial c_{i}(x, 0, z, t) / \partial y=0 \\
& D_{1 z} \partial c_{1}(x, y, 0, t) / \partial z-\beta_{z}\left(c_{1}(x, y, 0, t)-c_{0 z}(x, y)\right)=0 \\
& D_{i x} \partial c_{i}\left(L_{x}, y, z, t\right) / \partial x+\alpha_{i x}\left(c_{i}\left(L_{x}, y, z, t\right)-c_{i a x}(y, z)\right)=0, \\
& i=1 ; 2 \\
& D_{i y} \partial c_{i}\left(x, L_{y}, z, t\right) / \partial y+\alpha_{i y}\left(c\left(x, L_{y}, z, t\right)-c_{i a y}(x, z)\right)=0, \\
& i=1 ; 2 \\
& D_{2 z} \partial c_{2}\left(x, y, L_{z}, t\right) / \partial z+ \\
& \alpha_{z}\left(c_{2}\left(x, y, L_{z}, t\right)-c_{a z}(x, y)\right)=0 \\
& c_{1}\left(x, y, z_{1}, t\right)=c_{2}\left(x, y, z_{1}, t\right), \\
& D_{1 z} \partial c_{1}\left(x, y, z_{1}, t\right) / \partial z=D_{2 z} \partial c_{2}\left(x, y, z_{1}, t\right) / \partial z \\
& c_{i}(x, y, z, 0)=c_{i 0}(x, y, z), i=1 ; 2, \\
& x \in\left(0, L_{x}\right), y \in\left(0, L_{y}\right), z \in\left(0, L_{z}\right), \\
& t \in(0, t), i=1 ; 2
\end{aligned}
$$

where $c_{i}=c_{i}(x, y, z, t)$ are the concentrations functions in every layer, $f_{i}(x, y, z, t)$ - the fixed source function, $D_{i x}, D_{i y}, D_{i z}$ are constant diffusion coefficients, $\alpha_{i x}, \alpha_{i y}, \alpha_{z}, \beta_{z}, i=1 ; 2$ are the constant mass transfer coefficients for the 3rd type boundary conditions, $c_{a z}, c_{i a y}, c_{i a x}, c_{0 z}, i=1 ; 2$ are given concentration values on the boundaries, $t_{f}$ is the final time, $c_{i 0}(x, y, z), i=1 ; 2$ are given initial concentrations:

where $c_{i}=c_{i}(x, y, z, t)$ are the concentrations functions in every layer, $f_{i}(x, y, z, t)$ - the fixed source function, $D_{i x}, D_{i y}, D_{i z}$ are constant diffusion coefficients, $\alpha_{i x}, \alpha_{i y}, \alpha_{z}, \beta_{z}, i=1 ; 2$ are the constant mass transfer coefficients for the 3rd type boundary conditions, $c_{a z}, c_{i a y}, c_{i a x}, \quad c_{0 z}, i=1 ; 2$ are given concentration values on the boundaries, $t_{f}$ is the final time, $c_{i 0}(x, y, z), i=1 ; 2$ are given initial concentrations:

1) The homogenous 3rd type conditions by $x=L_{x}, y=L_{y}, z=L_{z}, z=0$,

2) The symmetrical conditions by $x=0, y=0$. The values $c_{i}$ and the flux functions $D_{i z} \partial c_{i} / \partial z$ must be continuous on the contact line between the layers $z=z_{1}$.

2. The averaged method in z-direction with the integral exponential spline

Using averaged method respect to $z$ with exponential or hyperbolic trigonometric functions we have

$c_{i}(x, y, z, t)=c_{i z}(x, y, t)+m_{i z} \frac{0.5 H_{i} \sinh \left(a_{i}\left(z-\bar{z}_{i}\right)\right)}{\sinh \left(0.5 a_{i} H_{i}\right)}$

$+e_{i z}\left(\frac{H_{i}^{2}}{4}\left(\frac{\sinh ^{2}\left(a_{i}\left(z-\bar{z}_{i}\right)\right.}{\sinh ^{2}\left(0.5 a_{i} H_{i}\right)}-A_{i 0 z}\right)\right)$,

where $c_{i z}(x, y, t)=H_{i}^{-1} \int_{z_{i-1}}^{z_{i}} c i(x, y, z, t) d z$,

$A_{i 0 z}=\frac{\sinh \left(a_{i} H_{i}\right) /\left(a_{i} H_{i}\right)-1}{\cosh \left(a_{i} H_{i}\right)-1}, \quad \bar{z}_{i}=\left(z_{i-1}+z_{i}\right) / 2$,

$z \in\left[z_{i-1}, z_{i}\right], i=1 ; 2$.

If parameters $a_{i}>0$ are tending to zero, then we obtain the limit as a parabolic spline because of $A_{i 0 z} \rightarrow 1 / 3$ [1]:

$c_{i}(x, y, z, t)=c_{i z}(x, y, t)+m_{i z}(x, y, t)\left(z-\bar{z}_{i}\right)+$

$e_{i z}(x, y, t)\left(\left(z-\bar{z}_{i}\right)^{2}-H_{i} / 12\right), i=1 ; 2$.

The unknown functions $m_{i z}(x, y, t), e_{i z}(x, y, t)$ it is possible to find out from the boundary conditions (2.1) in the following form in $z$-direction:

1) For $z=0$,

$d_{1}\left(m_{1 z}-e_{1 z} H_{1}\right)-$

$\beta_{z}\left(c_{1 z}-0.5 m_{1 z} H_{1}+e_{1 z} H_{1}\left(0.5 A_{11 z} H_{1}-c_{0 z}\right)\right)=0$,

2) For $z=L_{z}$, 
$d_{2}\left(m_{2 z}+e_{2 z} H_{2}\right)+$

$\alpha_{z}\left(c_{2 z}+0.5 m_{2 z} H_{2}+e_{2 z} H_{2}\left(0.5 A_{21 z} H_{2}-c_{a z}\right)\right)=0$,

3) For $z=z_{1}, c_{1 z}+0.5 m_{1 z} H_{1}+e_{1 z} H_{1} 0.5 A_{11 z} H_{1}=$ $c_{2 z}-0.5 m_{2 z} H_{2}+e_{2 z} H_{1} 0.5 A_{21 z} H_{2}$,

$d_{1 z}\left(m_{1 z}-e_{1 z} H_{1}\right)=d_{2 z}\left(m_{2 z}-e_{2 z} H_{2}\right)$,

where $d_{i z}=0.5 D_{i z} a_{i} \operatorname{coth}\left(0.5 a_{i} H_{i}\right)$,

$A_{i 1 z}=0.5\left(1-A_{i 0 z}\right) \rightarrow 1 / 3$, if $a_{i} \rightarrow 0, i=1 ; 2$.

From boundary conditions by $z=z_{1}$ follows:

$m_{i z}=m_{0 i}\left(c_{2 z}-c_{1 z}\right)+m_{i 1} e_{1 z} H_{1}+m_{i 2} e_{2 z} H_{2}$,

$i=1 ; 2$, where $m_{01}=2 d_{2 z} /\left(H_{2} s_{1}\right)$,

$m_{11}=-\left(d_{1 z}+A_{11 z} H_{1} d_{2 z} / H_{2}\right) / s_{1}$,

$m_{12}=d_{2 z}\left(A_{21 z}-1\right) / s_{1}, s_{1}=d_{1 z}+d_{2 z} H_{1} / H_{2}$,

$m_{02}=m_{01} d_{1 z} / d_{2}, m_{21}=\left(m_{11}+1\right) d_{1 z} / d_{2 z}$,

$m_{22}=m_{12} d_{1 z} /\left(d_{2 z}+1\right)$.

From boundary conditions by $z=0, z=L_{z}$ follows: $e_{1 z} H_{1} a_{11}+e_{2 z} H_{2} a_{12}=\beta_{z}\left(c_{1 z}-c_{0 z}\right)-b_{1}\left(c_{2 z}-c_{1 z}\right)$, $e_{1 z} H_{1} a_{21}+e_{2 z} H_{2} a_{22}=-\alpha_{z}\left(c_{2 z}-c_{a z}\right)-b_{2}\left(c_{2 z}-c_{1 z}\right)$, where $b_{1}=m_{01}\left(0.5 H_{1} \beta_{z}+d_{1 z}\right)$,

$b_{2}=m_{02}\left(0.5 H_{2} \alpha_{z}+d_{2 z}\right)$

$a_{11}=d_{1 z}\left(m_{11}-1\right)+0.5 \beta_{z} H_{1}\left(m_{11}-A_{11 z}\right)$,

$a_{22}=d_{2 z}\left(m_{22}+1\right)+0.5 \alpha_{z} H_{2}\left(m_{22}-A_{21 z}\right)$,

$a_{12}=d_{1 z} m_{12}+0.5 \beta_{z} H_{1} m_{12}$,

$a_{21}=d_{2 z} m_{21}+0.5 \alpha_{z} H_{2} m_{21}$.

$f_{i z}(x, y, t)=H_{i}^{-1} \int_{z_{i}-1}^{z_{i}} f_{i}(x, y, z, t) d z$,

$c_{i a x}^{v}(y)=H_{i}^{-1} \int_{z_{i}-1}^{z_{i}} c_{i a x}(y, z) d z$,

$c_{i a y}^{v}(x)=H_{i}^{-1} \int_{z_{i}-1}^{z_{i}} c_{i a y}(x, z) d z$,

$c_{i z, 0}^{v}(x, y)=H_{i}^{-1} \int_{z_{i}-1}^{z_{i}} c_{i 0}(x, y, z) d z, i=1 ; 2$.

3. The averaged method in y-direction

Using averaged method with respect to $y$ we have

$c_{i y}(x, t)=L_{y}^{-1} \int_{0}^{L_{y}} c_{i z}(x, y, t) d y$,

$c_{i z}(x, y, t)=c_{i y}(x, t)+$

$m_{i y}(x, t) \frac{0.5 L_{y} \sinh \left(a_{i}\left(y-0.5 L_{y}\right)\right)}{\sinh \left(0.5 a_{i} L_{y}\right)}+$

$e_{i y}(x, t)\left(\frac{L_{y}^{2}}{4}\left(\frac{\sinh ^{2}\left(a_{i}\left(y-0.5 L_{y}\right)\right.}{\sinh ^{2}\left(0.5 a_{i} L_{y}\right)}-A_{i 0 y}\right)\right)$,

With the unknown functions $m_{i y}(x, t), e_{i y}(x, t)$ we can determine these functions from the boundary conditions (3.1) in following form:
Then $e_{i z} H_{i}=c_{1 z} b_{i 1}+c_{2 z} b_{i 2}+c_{0 z} b_{i 3}+c_{0 z} b_{i 4}$, $i=1 ; 2$, where $b_{11}=\left(a_{22}\left(\beta_{z}+b_{1}\right)-b_{2} a_{12}\right) /$ det , $b_{12}=\left(a_{12}\left(\alpha_{z}+b_{2}\right)-b_{1} a_{22}\right) /$ det , $b_{21}=\left(-a_{21}\left(\beta_{z}+b_{1}\right)+b_{2} a_{11}\right) /$ det , $b_{22}=\left(-a_{11}\left(\alpha_{z}+b_{2}\right)+b_{1} a_{21}\right) /$ det, $b_{23}=a_{21} \beta_{z} /$ det,$b_{24}=a_{11} \alpha_{z} /$ det , $\operatorname{det}=a_{11} a_{22}+a_{12} a_{21}$.

Now the initial-boundary value $2 D$ problem is in following form

$$
\left\{\begin{array}{l}
\frac{\partial c_{i z}(x, y, t)}{\partial t}=\frac{\partial}{\partial x}\left(D_{i x} \frac{\partial c_{i z}(x, y, t)}{\partial x}\right)+ \\
\frac{\partial}{\partial y}\left(D_{i y} \frac{\partial c_{i z}(x, y, t)}{\partial y}\right)+f_{i z}(x, y, t)+(2.1) \\
B_{i z}\left(\begin{array}{l}
\left.c_{1 z}(x, y, t) b_{i 1}+c_{2 z}(x, y, t) b_{i 2}+\right) \\
c_{0 z}(x, y) b_{i 3}+c_{a z}(x, y) b_{i 4}
\end{array}\right) \\
x \in\left(0, L_{x}\right), y \in\left(0, L_{y}\right), t \in\left(0, t_{f}\right), \\
\partial c_{i z}(0, y, t) / \partial x=\partial c_{i z}(x, 0, t) / \partial y=0, \\
D_{i x} \frac{\partial c_{i z}\left(L_{x}, y, t\right)}{\partial x}+\alpha_{i x}\left(c_{i z}\left(L_{x}, y, t\right)-c_{i a x}^{v}(y)\right)=0, \\
D_{i y} \frac{\partial c_{i z}\left(x, L_{y}, t\right)}{\partial y}+\alpha_{i y}\left(c_{i z}\left(x, L_{y}, t\right)-c_{i a x}^{v}(x)\right)=0, \\
c_{i z}(x, y, 0)=c_{i z, 0}(x, y),
\end{array}\right.
$$

where

$$
B_{i z}=2 d_{i} / H_{i},
$$

$m_{i y}(x, t)=e_{i y}(x, t) L_{y}=-b_{i 5}\left(c_{i y}(x, t)-c_{i a y}^{v}(x)\right)$,

where $b_{i 5}=\alpha_{i y} /\left(0.5 L_{y} \alpha_{i y}\left(1+A_{i 1 y}\right)+2 d_{i y}\right)$,

$A_{i 1 y}=0.5\left(1-A_{i 0 y}\right)$,

$A_{\text {ioy }}=\frac{\sinh \left(a_{i} L_{y}\right) /\left(\left(a_{i} L_{y}\right)-1\right)}{\cosh \left(a_{i} L_{y}\right)-1}$,

$d_{i y}=0.5 L_{y} D_{i y} a_{i} \operatorname{coth}\left(0.5 a_{i} L_{y}\right)$.

Then the initial-boundary value problem (2.1) is in the following form

$$
\left\{\begin{array}{l}
\frac{\partial c_{i y}(x, t)}{\partial t}=\frac{\partial}{\partial x}\left(D_{i x} \frac{\partial c_{i y}(x, t)}{\partial x}\right)+f_{i y}(x, t)+ \\
B_{i y} b_{i 5} c_{i y}(x, t)+ \\
B_{i z}\left(c_{1 y}(x, t) b_{i 1}+c_{2 y}(x, t) b_{i 2}\right)+ \\
B_{i z}\left(c_{0 z}^{v}(x) b_{i 3}+c_{a z}^{v}(x) b_{i 4}\right)+B_{i y} b_{i 5} c_{i a y}^{v}(x), \\
x \in\left(0, L_{x}\right), t \in\left(0, t_{f}\right), \partial c_{i y}(0, t) / \partial x=0, \\
D_{i x} \partial c_{i y}\left(L_{x}, t\right) / \partial x+\alpha_{i x}\left(c_{i y}\left(L_{x}, t\right)-c_{i a x}^{v v}(y)\right)=0, \\
c_{i y}(x, 0)=c_{i y, 0}(x),
\end{array}\right.
$$

where $B_{i y}=2 D_{i y} / H_{i}$, 
$f_{i y}(x, t)=L_{y}^{-1} \int_{0}^{L_{y}} f_{i z}(x, y, t) d y$,

$c_{a z}^{v}(x)=L_{y}^{-1} \int_{0}^{L_{y}} c_{a z}(x, y) d y$,

$c_{0 z}^{v}(x)=L_{y}^{-1} \int_{0}^{L_{y}} c_{0 z}(x, y) d y$

$c_{i a x}^{v v}=L_{y}^{-1} \int_{0}^{L_{y}} c_{i a x}^{v}(y) d y$,

$c_{i y, 0}(x)=L_{y}^{-1} \int_{0}^{L_{y}} c_{i z, 0}(x, y) d y, i=1 ; 2$.

4. The averaged method in $x$-direction

It is possible to make the averaging also with respect to $x$

$c_{i x}(t)=L_{x}^{-1} \int_{0}^{L_{x}} c_{i y}(x, t) d x$,

$c_{i y}(x, t)=c_{i x}(t)+$

$m_{i x}(t) \frac{0.5 L_{x} \sinh \left(a_{i}\left(x-0.5 L_{x}\right)\right)}{\sinh \left(0.5 a_{i} L_{x}\right)}+$

$e_{i x}(t)\left(\frac{L_{x}^{2}}{4}\left(\frac{\sinh ^{2}\left(a_{i}\left(x-0.5 L_{x}\right)\right.}{\sinh ^{2}\left(0.5 a_{i} L_{x}\right)}-A_{i 0 x}\right)\right)$

with the unknown functions $m_{i x}(t), e_{i x}(t)$. We can determine these functions from the boundary conditions (4.1) in the following form:

$m_{i x}=e_{i x} L_{x}=-b_{i 6}\left(c_{i x}(t)-c_{i a x}^{v v}\right)$,

where $b_{i 6}=\alpha_{i x} /\left(0.5 L_{x} \alpha_{i x}\left(1+A_{i 1 x}\right)+2 d_{i x}\right)$.

$A_{i 1 x}=0.5\left(1-A_{i 0 x}\right)$,

$A_{\text {iox }}=\frac{\sinh \left(a_{i} L_{x}\right) /\left(\left(a_{i} L_{x}\right)-1\right)}{\cosh \left(a_{i} L_{x}\right)-1}$.

Then the initial-boundary value problem (3.1) is in following form

$$
\left\{\begin{array}{l}
\frac{\partial c_{i x}(t)}{\partial t}=-\left(B_{i x} b_{i 6}+B_{i y} b_{i 5}\right) c_{i x}(t)+ \\
f_{i x}(t)+B_{i z}\left(b_{i 1} c_{1 x}(t)+b_{i 2} c_{2 x}(t)\right)+ \\
B_{i z}\left(c_{0 z}^{v v} b_{i 3}+b_{i 4} c_{a z}^{v v}\right)+ \\
B_{i y} b_{i 5} c_{i a y}^{v v}+B_{i x} b_{i 6} c_{i a x}^{v v}, t \in\left(0, t_{f}\right), \\
c_{i x}(0)=c_{i x, 0}
\end{array}\right.
$$

where

$$
f_{i x}(t)=L_{x}^{-1} \int_{0}^{L_{x}} f_{i y}(x, t) d x,
$$

$c_{a z}^{v v}=L_{x}^{-1} \int_{0}^{L_{x}} c_{a z}^{v}(x) d x, c_{i x, 0}=L_{x}^{-1} \int_{0}^{L_{x}} c_{i y, 0}(x) d x$,

$c_{0 z}^{\nu v}=L_{x}^{-1} \int_{0}^{L_{x}} c_{0 z}^{v}(x) d x$

Therefore we have from (4.1) the initial problem for ODEs of the first order:

$$
\begin{aligned}
& \left\{\begin{array}{l}
\dot{u}_{1}(t)=c_{11} u_{1}(t)+c_{12} u_{2}(t)+e_{1}(t), \\
\dot{u}_{2}(t)=c_{21} u_{1}(t)+c_{22} u_{2}(t)+e_{2}(t),(4.2) \\
u_{1}(0)=u_{10}, u_{2}(0)=u_{20},
\end{array} \quad u_{i}(t)=c_{i x}(t), \quad \dot{u}_{i}=\frac{\partial u_{i}(t)}{\partial t},\right. \\
& \text { where } \quad \\
& u_{i}(0)=c_{i x, 0}, i=1 ; 2, \\
& e_{i}(t)=B_{i z}\left(c_{0 z}^{v v} b_{i 3}+b_{i 4} c_{a z}^{v v}\right)+B_{i y} b_{i 5} c_{i a y}^{v v}+B_{i x} b_{i 6} c_{i a x}^{v v} \\
& +f_{i x}(t), \\
& c_{11}=B_{1 z} b_{11}-\left(B_{1 x} b_{16}+B_{1 y} b_{15}\right), \\
& c_{22}=B_{2 z} b_{22}-\left(B_{2 x} b_{26}+B_{2 y} b_{25}\right), \\
& c_{21}=B_{2 z} b_{21}, c_{12}=B_{1 z} b_{12} .
\end{aligned}
$$

The solutions of (4.2) can be obtained with the help of classical methods [6]. For the averaged stationary solution follows the analytical solution in the form $c_{1 x}=\left(e_{2} c_{12}-e_{1} c_{22}\right) / d, c_{2 x}=\left(e_{1} c_{21}-e_{2} c_{11}\right) / d$, where $d=c_{11} c_{22}-c_{12} c_{21}$.

5. Analytical model for estimating the parameters $a_{1}, a_{2}$

We consider the special 1-D diffusion problem in the $z$ - direction for $f=\alpha_{x}=\alpha_{y}=0$,

$c_{a z}(x, y)=C_{a} \cos \left(\pi x / L_{x}\right) \cos \left(\pi y / L_{y}\right)$, $c_{0 z}(x, y)=C_{0} \cos \left(\pi x / L_{x}\right) \cos \left(\pi y / L_{y}\right)$. Then the stationary solution of (2.1) is in the form $c_{i}(x, y, z)=c_{i}(z) \cos \left(\pi x / L_{i x}\right) \cos \left(\pi y / L_{i y}\right)$, where the function $c_{i}(z)$ is solution for following boundaryvalue problem:

$$
\left\{\begin{array}{l}
\frac{\partial}{\partial z}\left(\frac{\partial c_{i}(z)}{\partial z}\right)-b_{i}^{2} c_{i}(z)=0, z \in\left(z_{i-1}, z_{i}\right), \\
D_{1 z} \partial c_{1}(0) / \partial z-\beta_{z}\left(c_{1}(0)-C_{0}\right)=0, \\
D_{2 z} \partial c_{2}\left(L_{z}\right) / \partial z+\alpha_{z}\left(c_{2}\left(L_{z}\right)-C_{a}\right)=0 \\
c_{1}\left(H_{1}\right)=c_{2}\left(H_{2}\right), D_{1 z} \frac{\partial c_{1}\left(H_{1}\right)}{\partial z}=D_{2 z} \frac{\partial c_{2}\left(H_{1}\right)}{\partial z}
\end{array},\right.
$$

where $b_{i}=\pi \sqrt{\left(D_{i x} / L_{x}^{2}+D_{i y} / L_{y}^{2}\right) / D_{i z}}, i=1 ; 2$.

Therefore the exact solution is

$c_{1}(z)=P_{1} \sinh \left(b_{1} z\right)+P_{2} \cosh \left(b_{1} z\right)$, $c_{2}(z)=P_{3} \sinh \left(b_{2} z\right)+P_{4} \cosh \left(b_{2} z\right)$, where the constants $P_{1}, P_{2}, P_{3}, P_{4}$ are the functions of $D_{i x}, D_{i y}, D_{z}, L_{x}, L_{y}, L_{z}, \alpha_{z}, \beta_{z}, C_{0}, C_{a}$. The averaged values are $c_{1}^{v}=H_{1}^{-1} \int_{0}^{H_{1}} c_{1}(z) d z=\left(H_{1}\right)^{-1} \frac{P_{1}}{b_{1}}\left(\cosh \left(b_{1} H_{1}\right)-1\right)$ $+\left(H_{1}\right)^{-1} \frac{P_{2}}{b_{1}} \sinh \left(b_{1} H_{1}\right)$, 
$c_{2}^{v}=H_{2}^{-1} \int_{H_{1}}^{L_{z}} c_{2}(z) d z=$

$\left(H_{2}\right)^{-1}\left(\frac{P_{3}}{b_{2}}\left(\cosh \left(b_{2} L_{z}\right)-\cosh \left(b_{2} H_{1}\right)\right)\right)+$

$\left(H_{2}\right)^{-1}\left(\frac{P_{4}}{b_{2}}\left(\sinh \left(b_{2} L_{z}\right)-\sinh \left(b_{2} H_{1}-1\right)\right)\right)$.

This form of solution remained also for discrete approximation $c\left(z_{j}\right)=C_{j}, z_{j}=(j-1) h, \quad h=\frac{L_{z}}{N}$, $j=\overline{1, N P}, H_{1}=h N 1$ by using exact finite diference scheme (FDS) from N.Bahvalov [2]:

$c_{j}=P_{1} \sinh \left(b_{1} z_{j}\right)+P_{2} \cosh \left(b_{1} z_{j}\right), j=\overline{1, N 1}$,

$c_{j}=P_{3} \sinh \left(b_{2} z_{j}\right)+P_{4} \cosh \left(b_{2} z_{j}\right)$,

$j=\overline{N 1, N 1+1}$.

1) $C_{2}-C_{1}\left(1+h \frac{\beta_{z}}{D_{1 z}}\right)+h C_{0} \frac{\beta_{z}}{D_{1 z}}=0, z=z_{1}=0$,

2) $C_{N}-C_{N+1}\left(1+h \frac{\alpha_{z}}{D_{2 z}}\right)+h C_{a} \frac{\alpha_{z}}{D_{2 z}}=0$,

$z=z_{N+1}=L_{z}$,

3) $\begin{aligned} & P_{1} \sinh \left(b_{1} H_{1}\right)+P_{2} \cosh \left(b_{1} H_{1}\right)=\text {, for } \\ & P_{3} \sinh \left(b_{2} H_{1}\right)+P_{4} \cosh \left(b_{1} H_{1}\right)\end{aligned}$,

$z=H_{1}, j=N 1$,

4) $\frac{D_{1 z}\left(C_{N 1}-C_{N 1-1}\right)}{h}=\frac{D_{2 z}\left(C_{N 1+1}-C_{N 1}\right)}{h}$,

for $z=H_{1}, j=N 1$.

Coefficients $P_{1}, P_{2}, P_{3}, P_{4}$ are obtained from:

$P_{1}=b_{6} P_{2}-b_{5} C_{0}, P_{3}=b_{4} P_{4}-b_{3} C_{a}$,

$b_{3}, b_{4}, b_{5}, b_{6}$ are the functions $g_{i}$ dependent on a variety of variables:

$b_{3}=g_{1}\left(h, \alpha_{z}, D_{2 z}\right), b_{4}=g_{2}\left(h, \alpha_{z}, D_{2 z}, b_{2}, L_{z}\right)$,

$b_{5}=g_{3}\left(h, \beta_{z}, D_{1 z}, b_{1}\right), b_{6}=g_{4}\left(h, \beta_{z}, D_{2 z}, b_{1}\right)$.

Coefficients $P_{2}, P_{4}$ are the functions $g_{5}, g_{6}$ dependent of variables

$C_{0}, C_{a}, H_{1}, H_{1}+h, H_{1}-h$,

$\alpha_{z}, \beta_{z}, D_{1 z}, D_{2 z}, b_{1}, b_{2}, L_{z}$.

For comparing we use the averaged method with respect to $z$ with exponentials spline. Then

$\alpha_{i x}=\alpha_{i y}=0, c_{i z}=c_{i x}$,

$e_{i}=B_{i z}\left(C_{0} b_{i 3}+C_{a} b_{i 4}\right), i=1 ; 2$,

$c_{11}=B_{1 z} b_{11}-D_{1 z} b_{1}^{2}, c_{12}=B_{1 z} b_{12}, c_{21}=B_{2 z} b_{21}$,

$c_{22}=B_{2 z} b_{22}-D_{2 z} b_{2}^{2}$, and using the averaged method in $z$-direction the functions $c_{a z}, c_{0 z}$ are replaced with $C_{a}, C_{0}$.

We have following numerical results ( $L_{z}=1, H_{1}=0.6, H_{2}-0.4, L_{x}=L_{y}=1, \quad C_{0}=0.3$,

$C_{a}=2.0, D_{1 z}=10^{-3}, \alpha_{z}=20$,

( $a_{1}=a_{2}=0.0001 \approx 0$ - for parabolic spline).

$D_{1 x}=D_{1 y}=10^{-4}, \quad b_{1}=1.4050, \quad \beta_{z}=10$, $b_{2}=4.4429, D_{2 z}=5 \cdot 10^{-4}, D_{2 x}=D_{2 y}=5 \cdot 10^{-4}$ ) for maximal error $\delta$ and averaged values $c_{1 z}, c_{2 z}$ depending on $a_{1}, a_{2}\left(c_{1}^{v}=0.3558, c_{2}^{v}=0.9809\right)$. The numerical results are given in the TABLE 1

The solution $c_{1 z}, z \in[0,0.6], c_{2 z}, z \in[0.6,1.0]$ for 3 methods $(N=20)$ is represented in Figs. 1, 2. For FDS we have $\delta=0.0336(N=20), 0.0160(N=40)$, $0.0078(N=80)$.

\section{TABLE 1}

THE MAXIMAl ERror $\partial$ AND AVERAged VAlues $c_{1 z}, c_{2 z}$ DEPENDING ON $a_{1}, a_{2}$

\begin{tabular}{|l|l|l|l|l|}
\hline \multicolumn{1}{|c|}{$a_{1}$} & \multicolumn{1}{c|}{$a_{2}$} & $\partial$ & $c_{1 z}$ & $c_{2 z}$ \\
\hline 0 & 0 & 0.1252 & 0.3022 & 0.9263 \\
\hline 0.5 & 0.5 & 0.1235 & 0.3030 & 0.9273 \\
\hline 1 & 1 & 0.1185 & 0.3051 & 0.9304 \\
\hline 2 & 2 & 0.0996 & 0.3132 & 0.9421 \\
\hline 3 & 3 & 0.0710 & 0.3250 & 0.9597 \\
\hline 4 & 4 & 0.0450 & 0.3390 & 0.9812 \\
\hline 5 & 5 & 0.0528 & 0.3528 & 1.0046 \\
\hline 3 & 4 & 0.0378 & 0.3408 & 0.9842 \\
\hline 2 & 4 & 0.0316 & 0.3422 & 0.9867 \\
\hline 1 & 4 & 0.0283 & 0.3431 & 0.9884 \\
\hline 1.2 & 4 & 0.0281 & 0.3430 & 0.9882 \\
\hline
\end{tabular}

Using averaged method with respect to $z$ with 2 unknown parameters in every layer we have

$c_{i}(x, y, z, t)=c_{i z}(x, y, t)+m_{i z} \frac{0.5 H_{i} \sinh \left(a_{i}\left(z-\bar{z}_{i}\right)\right)}{\sinh \left(0.5 a_{i} H_{i}\right)}$

$+e_{i z}\left(\frac{H_{i}^{2}}{4}\left(\frac{\sinh ^{2}\left(a_{0 i}\left(z-\bar{z}_{i}\right)\right.}{\sinh ^{2}\left(0.5 a_{0 i} H_{i}\right)}-A_{i 0 z}\right)\right)$,

$A_{i 0 z}=\frac{\sinh \left(a_{0 i} H_{i}\right) /\left(a_{i} H_{i}\right)-1}{\cosh \left(a_{0 i} H_{i}\right)-1}, i=1 ; 2$.

We have the exact solution for $a_{1}=b_{1}, a_{2}=b_{2}$, $a_{01}=\frac{b_{1}}{2}, a_{02}=\frac{b_{2}}{2}$ for every other parameters. $\alpha, \beta, C_{0}, C_{a}, H_{1}, H_{2}, L_{z}, D_{1 z}, D_{2 z}$, $b_{1}, b_{2}$. 


\section{RESULTS AND DISCUSSION}

6. Some numerical results

A uniform grid in the space $((M+1) \times(N+1))$ : $\left\{\left(y_{i}, x_{j}\right), y_{i}=(i-1) h y x_{j}=(j-1) h x\right\}$,

$i=\overline{1, M+1}, j=\overline{1, N+1}, \quad M h y=L_{y}, N h x=L_{x}$ with a time $t$ moments $t_{n}=n \tau, n=0,1, \ldots$. Subscripts $(i, j, n)$ refer to $y, x, t$ indices with the mesh spacing and for approximation the functions $c_{k z}(x, y, t), k=1 ; 2$ we have the grid function with following values: $U 1_{i, j}^{n} \approx c_{1 z}\left(x_{j}, y_{i}, t_{n}\right)$, $U 2_{i, j}^{n} \approx c_{2 z}\left(x_{j}, y_{i}, t_{n}\right) \quad$ or $\quad U k_{i, j}^{n} \approx c_{k z}\left(x_{j}, y_{i}, t_{n}\right)$, $k=1 ; 2$.

For grid function's $U k_{i, j}^{n}$ calculation Tomas algorithm in $x$ and $y$ directions was used for realization the alternating direction method (ADI) of Douglas and Rachford (1955) and 3-point difference equations for every direction were constructed. The numerical results are obtained for [5]:

$z_{m}=m h_{z}, m=\overline{0,30}, h_{z}=\frac{L_{z}}{30}$,

$D_{1 x}=D_{1 y}=10^{-4}, D_{2 x}=D_{2 y}=5 \cdot 10^{-4}$,

$L_{z}=3, a_{1}=4.5, a_{2}=3.0$,

$H_{2}=1.2, L_{x}=L_{y}=1 \alpha_{z}=20, \beta_{z}=10$,

$\alpha_{1 x}=2, \alpha_{2 x}=2, \alpha_{1 y}=4, \alpha_{2 y}=4$,

$c_{1 a x}^{v}=2.5, c_{2 a x}^{v}=2.5, c_{1 a y}^{v}=2.5, c_{2 a y}^{v}=2.5$,

$N=22, M=20, a_{1}=4.5, a_{2}=3.0$.

On the top of earth $\left(z=L_{z}\right)$ the concentration $c[\mathrm{mg} / \mathrm{kg}]$ of metals is measured in following nine points in the $(x, y)$ plane:

$c(0.1,0.2)=3.69 ;$
$c(0.9,0.2)=3.72 ;$
$c(0.5,0.5)=4.63 ;$
$c(0.1,0.8)=3.71 ;$
$c(0.9,0.8)=3.73$.

$c(0.5,0.2)=4.43$

$c(0.1,0.5)=4.00$;

$c(0.9,0.5)=4.11$;

$c(0.5,0.8)=4.50$

This date are smoothing in matrix $c_{a z}$ by $2 \mathrm{D}$ interpolation with MATLAB operator, using the spline function. In Fig. 2 we can see the distribution of concentration $c$ for Ca in the $(x, y)$ plane by $z=L_{z}$. On the below of peat block $z=0$ the elements of matrix $c_{0 z}$ have a constant value $1.30 \mathrm{mg} / \mathrm{kg}$.

For the initial condition the averaged solutions $c_{k z}(x, y), k=1 ; 2$ are chosen. We have the stationary solution with $\tau=10, t_{f}=200$ the maximal error $10^{-6}$, the maximal value of $c_{1 z}(x, y) 2.500$ for averaged method and for ADI method (following results we can see in Figs 3, 4.).

Depending on the number of the grid points $(N, M)$ we have following maximal values for averaged method and ADI methods:

$4.6311 \quad(N=22, \quad M=20), \quad 4.6298 \quad(N=12$, $M=10)$, $4.6324(N=32, M=30)$.

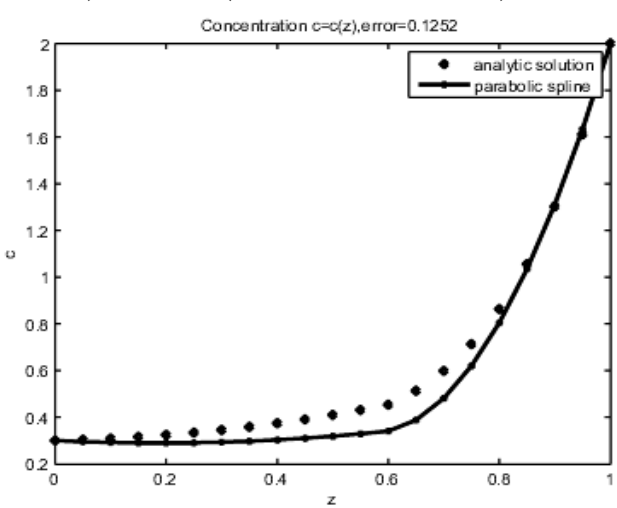

Fig. 1. Solution $c_{1}(z), c_{2}(z)$ for $\alpha_{z}=20, \beta_{z}=10$ $a_{1}=1.2, a_{2}=4.0$, using parabolic spline.

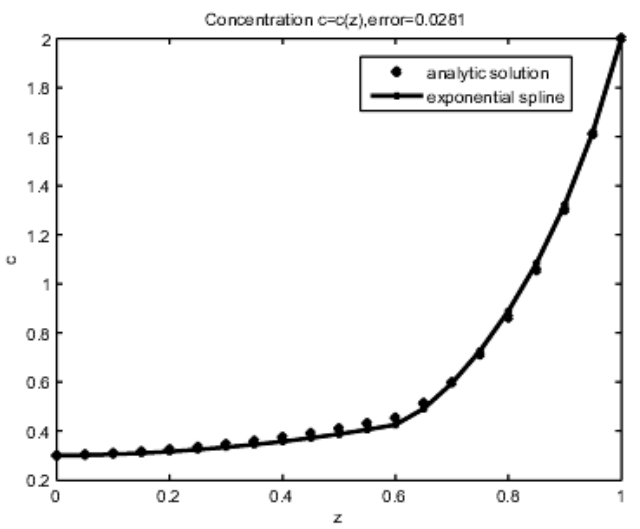

Fig. 2. Solution $c_{1}(z), c_{2}(z)$ for $\alpha_{z}=20, \beta_{z}=10$ $a_{1}=1.2, a_{2}=4.0$, using exponential spline.

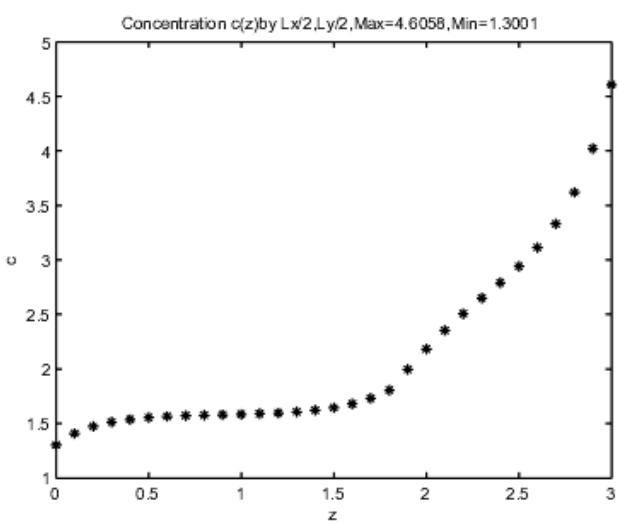

Fig. 3. Graphic of averaged concentration $c(z)$ for $x=L_{x} / 2, y=L_{y} / 2$ 


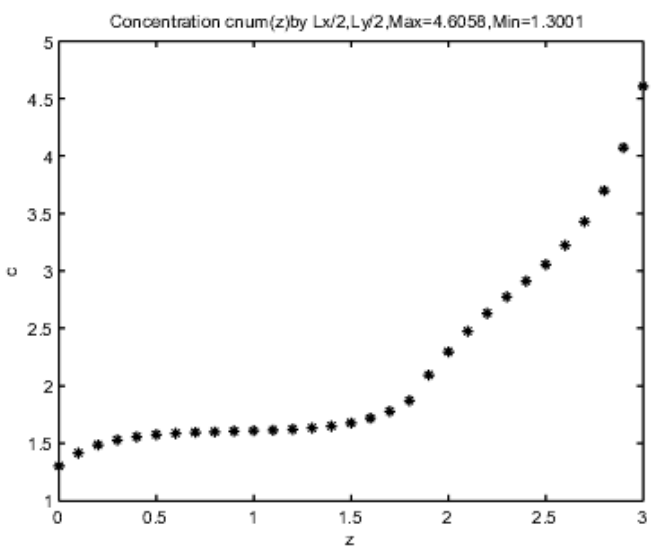

Fig. 4. Graphic of numerical concentration $c(z)$ for

$$
x=L_{x} / 2, y=L_{y} / 2 \text {. }
$$

\section{CONCLUSIONS}

The calculations were carried out with the mathematical model, was confirmed by the results of the measurements carried out on the surface of the Earth and the deeper layers - the highest concentrations of metals are at the top surface of the Earth, then its very rapidly decreases with increasing depth of peat layer.

Elements concentration in peat profiles confirms with respect to the possibility of using trace elements concentration as an indicator of the region and global environmental pollution.

The 3-D diffusion problem in peats block is reduced to 2-D and 1-D problems used the integral parabolic and exponential splines. The 1-D differential and discrete problems are solved analytically.
Increasing the number of parameters from one to two for the exponential spline formula, in the process of solving one dimension task the exact solution for calculating the concentration in $\mathrm{z}$-direction was obtained.

The solutions of corresponding averaged non stationary 2-D initial-boundary value problem are obtained numerically using alternating-direction implicit (ADI) method of Douglas and Rachford. The numerical solution is compared with the designed analytical solution.

The relative error in the calculation of the concentration of heavy metals (FE, CA) with the averaged method which has been designed by authors, and the grid method does not exceed $2 \%-3 \%$. It is perfectly acceptable (valid for use) accuracy to tackle practical engineering technical problems.

\section{REFERENCES}

[1] A. Buikis. The analysis of schemes for the modelling same processes of filtration in the underground. Riga, Acta Universitatis Latviensis, 592 1994, pp. 25-32 (in Latvian).

[2] N. S. Bahvalov, N. P. Zhitkov, G. M. Kobelhkov. Numerical mthods. M:, Nauka, 1987 (in Russian).

[3] A. Buikis. The approximation with splines for problems in layered systems. Riga, Acta Universitatis Latviensis, 592 1994, pp. 135-138 (in Latvian).

[4] I. Kangro, H. Kalis, A. Gedroics, E. Teirumnieka, E. Teirumnieks. On mathematical modllingof metals distributions in peat layers. Mathematical Modelling and Analysis, 19:4 2014, pp. 568-588. Taylor \& Francis, Mortimer House, 37-41, London W1T 3JH, UK.

[5] E. Teirumnieka, E. Teirumnieks, I. Kangro, H. Kalis, A. Gedroics. The mathematical modeling of $\mathrm{Ca}$ and $\mathrm{Fe}$ distribution in peat layers. Proceed. of the 8-th int. scientific practical conference "Environment. Technology. Resources.", pp. 40-47, Rezekne higher education institution, June 20-22, 2 2011.

[6] C. Henry Edwards, David E. Penny. Differential equations and boundary value problems, computing and modelling. Pearson, Prentice Hal, third edition, 2008 (in Russian). 